\title{
Melanocitoma do nervo óptico
}

\section{Optic disk melanocytoma: bibliographic review}

Enéias Bezerra Gouveia', Maira Saad de Ávila Morales²

\section{ResUMO}

O melanocitoma do disco óptico é bem conhecido como uma variante do nervo melanocítico que geralmente ocorre no disco óptico. Historicamente o tumor foi confundido com melanoma maligno. Histopatologicamente é composto por células redondas densamente pigmentadas com citoplasma abundante e pequenos nucléolos. $\mathrm{O}$ melanocitoma é considerado um tumor benigno, estacionário e com pouca predisposição de sofrer malignação. Na maioria dos casos são assintomáticos, mas podem apresentar defeitos pupilares e no campo visual. É importante salientar que a transformação maligna em melanoma ocorre em 1 a $2 \%$ dos casos. Assim, os oftalmologistas devem familiarizar-se com o melanocitoma do disco óptico e os pacientes afetados devem ser acompanhados periodicamente.

Descritores: Melanoma/diagnóstico; Disco óptico/patologia; Neoplasias do nervo óptico/ pathology; Melanócitos/patologia; Diagnóstico diferencial; Revisão

\footnotetext{
'Oftalmologista do Centro Médico Avimed - São Paulo (SP), Brasil;

²Mestre, médica colaboradora da Escola Paulista de Medicina da Universidade Federal de São Paulo - UNIFESP - São Paulo (SP), Brasil.
} 


\section{INTRODUÇÃO}

$\mathbf{0}$ melanocitoma do disco óptico apresenta-se como uma lesão densamente pigmentada, pouco sobrelevada, arredondada, variando do cinza ao preto e com diversos tamanhos. Representa uma variante específica do nervo melanocítico uveal.

Existem relatos de malignização em casos de melanocitomas. O crescimento da lesão com espessamento local deve servir de alerta, sugerindo uma possível transformação maligna ${ }^{(1.3)}$.

Historicamente a possibilidade de malignização fez da enucleação o modo preferido de tratamento desta neoplasia, porém nas últimas décadas o tratamento sofreu mudanças para simples observação seriada.

Este artigo objetivou rever dados históricos, prevalência, patogênese, histopatologia, transformação maligna, quadro clínico, diagnóstico diferencial, exames complementares e conduta do tumor.

\section{Histórico}

Antes dos trabalhos pioneiros de Zimmerman ${ }^{(4-6)}$ as lesões densamente pigmentadas do disco óptico eram consideradas como melanoma maligno primário do disco óptico. Loewenstein sugeriu que tais lesões poderiam representar uma transformação maligna do nevus do disco óptico ${ }^{(4-6)}$.Como resultados de um estudo clínico e histopatológico, realizado por um longo período de acompanhamento, Zimmerman instituiu o termo "melanocitoma", para indicar o comportamento benigno desta lesão pigmentada do disco óptico ${ }^{(4-6)}$.

\section{Prevalência}

A prevalência exata do melanocitoma é difícil de estimar devido ser um achado ocasional de exame de rotina, bem como por ter sido confundido por muitos anos clínica e histopatologicamente como melanoma maligno. Além do fato de poucos pacientes procurarrem o oftalmologista por ser assintomático em sua grande maioria e ter uma lenta deteriorização visual e podendo causar defeito de campo visual ${ }^{(8)}$.

O melanocitoma apresenta leve predileção pelo sexo feminino ${ }^{(4)}$ e por indivíduos de raças pigmentadas, sendo raras em brancos ${ }^{(4)}$, no entanto não há uma explicação aparente para esta predisposição de sexo e raça. A idade média do diagnóstico é de 50 anos, variando de $1-91 \operatorname{anos}^{(9)}$.

\section{Patogênese}

A patogênese do melanocitoma do disco óptico é desconhecida. É possível que o melanocitoma seja uma lesão congênita, mas que só se torna clinicamente aparente em uma fase tardia da vida, talvez devido à aquisição de pigmentos em uma lesão previamente amelanótica ${ }^{(1)}$. Existem relatos de desenvolvimento em adultos com documentação fotográfica prévia como normal, abrindo especulação para existência de uma forma adquirida ou também para uma apresentação de uma lesão amelanótica que se torna pigmentada ${ }^{(10)}$.

\section{Histopatologia}

O melanocitoma descreve um tumor pigmentado do nervo óptico, podendo acometer também a íris ${ }^{(11)}$, coróide $^{(12)}$,esclera ${ }^{(13)}$, conjuntiva ${ }^{(14)}$ corpo ciliar ${ }^{(15-16)}$. A maioria das células pigmentadas intra-ocular tem origem nas células pluripotentes da crista neural, nestes se incluem nevo, melanomas e melanocitomas. O melanocitoma é considerado uma forma especial do nevo uveal, podendo ocorrer em qualquer local que apresente melanócitos. Histologicamente as células se apresentam intensamente pigmentadas por melanina, são grandes, poliédricas, com abundante citoplasma contendo núcleos pequenos e nucléolos pequenos ou ausentes ${ }^{(17-20)}$. O tumor freqüentemente envolve as camadas de fibras nervosas da retina adjacente e demonstra margem fibrilada. Na maioria dos casos, os melanocitomas são contíguos com a coróide justapapilar. Juarez e Tso ${ }^{(21)}$, através de estudo com microscopia eletrônica, identificaram dois tipos celulares de melanocitoma. No tipo I, as células são poliédricas, apresentam melanossomos gigantes e poucas organelas citoplasmáticas. No tipo II, as células são pequenas, fusiformes, contêm nucléolos proeminentes, numerosas mitocôndrias, ribossomos e retículo endoplasmático.

\section{Transformação maligna}

O melanocitoma é um tumor benigno, estacionário, com pouco potencial de crescimento, bem como quase nenhuma propensão de sofrer transformação maligna, que é considerada uma complicação rara ${ }^{(1.39 \cdot 922)}$, apesar de que, nos últimos anos, têm sido registrados alguns casos de transformação maligna provada histologicamente ${ }^{(23-24)}$. Esta mudança maligna é estimada ocorrer em aproximadamente 1 a $2 \%$ dos $\operatorname{casos}^{(9)}$. Comprometimento extenso do nervo óptico com importante baixa da acuidade visual é sugestivo de transformação maligna ${ }^{(92-2526)}$. Nestes casos, a evidência de transformação maligna, em particular o aumento em seu tamanho e achados ecográficos correlacionados, necessita de uma avaliação precisa com o objetivo de se detectar o mais precocemente possível as alterações compatíveis com melanoma maligno a fim de se instituir terapêutica adequada ${ }^{(22)}$. 


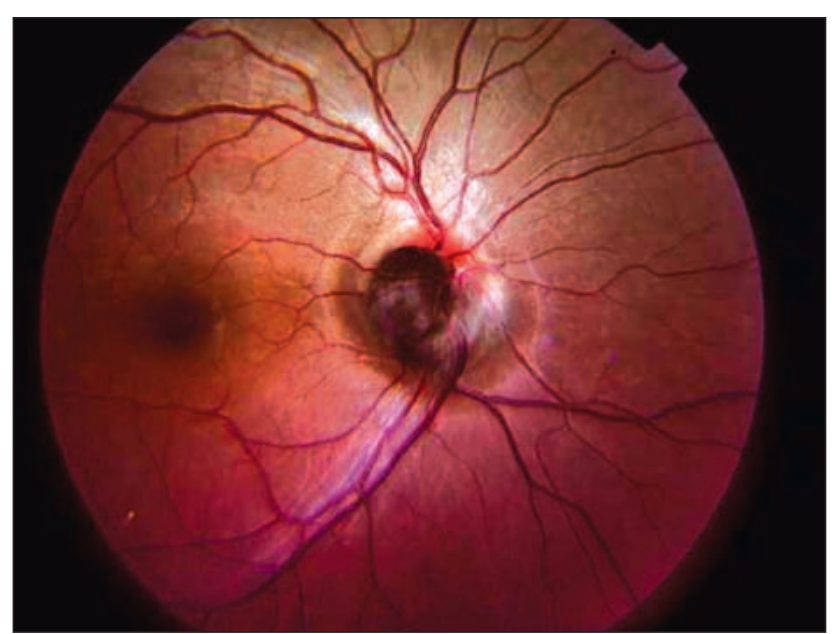

Figura 1: Retinografia - Observou-se lesão pigmentada, sobrelevada e arredondada, no quadrante temporal inferior do disco óptico do olho direito

\section{Quadro clínico}

Raramente existe queixa oftalmológica objetiva. A baixa de acuidade visual é uma forma rara de apresentação cujos fatores de riscos incluem extensão tumoral e a presença de fluido sub-retiniano ${ }^{(9)}$.

A anisocoria também é bastante rara nestes pacientes, no entanto o defeito pupilar aferente acontece em pelo menos $30 \%$ dos pacientes com melanocitoma do disco óptico, de provável origem compressiva $^{(8-27)}$.

Oftalmoscopicamente a apresentação do melanocitoma do disco óptico varia de caso para caso, embora classicamente seja visto como uma lesão elevada, freqüentemente no quadrante temporal inferior do disco óptico, variando do cinza ao preto, de tamanho variável (Figura 1). Em 60\% dos casos não excedem um diâmetro de disco óptico, contudo há registro de uma lesão de tamanho de seis diâmetros de papila ${ }^{(26)}$. Joffe et al. ${ }^{(26)}$ avaliaram diversos tipos de apresentação clínica do melanocitoma em 40 pacientes. Eles encontraram a lesão confinada à cabeça do nervo óptico em $18 \%$ dos pacientes, estendendo nas camadas de fibras nervosas da retina em $77 \%$, apresentando um componente coroidal justapapilar em 47\% e demonstrou mínimo crescimento em 15\%. Em 75\% dos olhos envolvidos, a acuidade visual variou de $20 /$ 15 a $20 / 30$.

Foram relatados alguns casos raros de associações oculares, embora consideradas prováveis coincidências, tais como melanose racial, hipoplasia do disco óptico, retinose pigmentar, hipertrofia congênita do epitélio pigmentar da retina, neurofibromatose tipo dois, carcinoma basocelular ${ }^{(9-28)}$.

\section{Diagnóstico diferencial}

Diante de um quadro de suspeita de melanocitoma, devemos fazer diagnóstico diferencial com nevo peripapilar, hamartomas combinado da retina com epitélio pigmentar da retina (EPR), hiperplasia do EPR, adenoma do EPR, melanoma metastático do disco óptico e melanoma maligno ${ }^{(18-29-30)}$, sendo este a principal entidade diagnóstica diferencial devido a sua morbidade e mortalidade.

O Nevo de coróide é uma lesão coroidal plana, ou de mínima elevação que encontra-se fora do disco óptico e não sobre ele. Contudo, muitos melanocitomas têm componente justapapilar que é idêntico ao nevo de coróide, bem como têm sido observados casos de nevo justapapilar com pequeno componente no disco óptico, tornando-se difícil a classificação primária entre nevo de coróide ou melanocitoma do nervo óptico ${ }^{(19)}$.

O Hamartoma justa papilar da retina e EPR podem ser muito parecidos com o melanocitoma, embora geralmente não envolva o disco óptico em si, mas estende-se de sua margem. Está associado com gliose, que pode causar tração nos vasos retinianos tornando-os retificados ${ }^{(31)}$.

A Hiperplasia do EPR por sua vez ocorre após trauma ocular, processo inflamatório ou intervenção cirúrgica ${ }^{(19)}$.

O Adenoma do EPR justapapilar também pode lembrar o melanocitoma, embora possa se estender da margem do disco óptico não apresenta margem fibrilada ${ }^{(32)}$. Em contraste com o melanocitoma ele pode ocasionalmente ser amelanótico ${ }^{(33)}$.

O Melanoma cutâneo mestastático para o disco óptico é extremamente raro. No entanto, seu crescimento é rápido e infiltra difusamente o disco óptico, configurando um papiledema ou papilite aguda ${ }^{(34)}$.

O Melanoma maligno do disco óptico é originado do tecido coroidal justapapilar que espalha sobre o disco, sendo extremamente raro sua confinação no nervo óptico ${ }^{(35-36)}$. Trata-se do tumor intra-ocular mais comum entre adultos acima dos 50 anos de idade e com maior incidência na raça branca. Oftalmoscopicamente estes tumores, em geral são mais largos, crescem mais rápido e são tipicamente amarelado ou marrom em comparação ao melanocitoma. Apresenta ecograficamente refletividade média para baixa, presença de sinais de vascularidade, além do ângulo kappa ${ }^{(17)}$. Na angiofluoresceinografia destes pacientes evidencia uma hiperfluorescência durante todo o estudo ${ }^{(37)}$. $\mathrm{Na}$ tomografia de coerência óptica o melanoma se caracteriza por uma camada de alta refletividade devido ao EPR e a coriocapilar atenuada pelos sinais coroidianos 


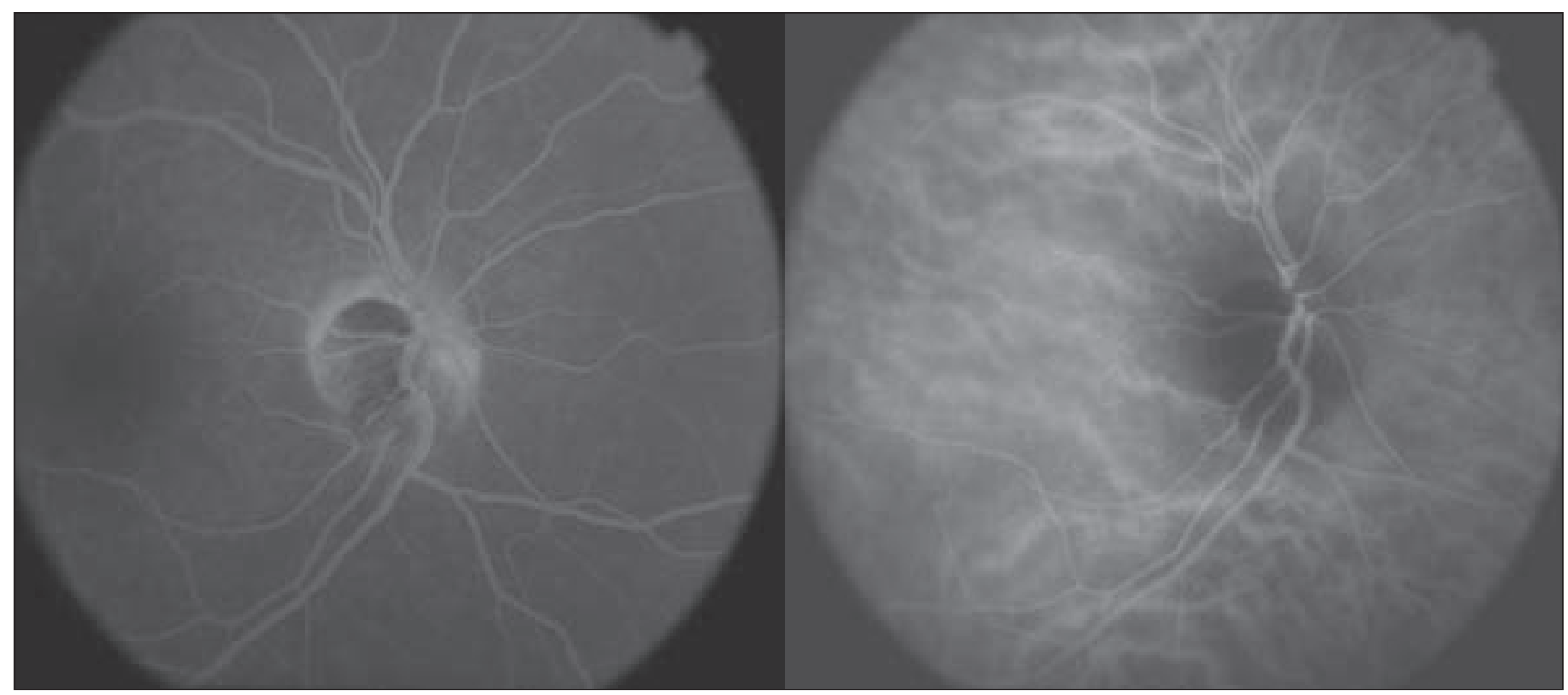

Figura 2: Angiofluoresceinografia do melanocitoma do disco óptico, com a característica hipofluorescência que se mantém ao longo do estudo

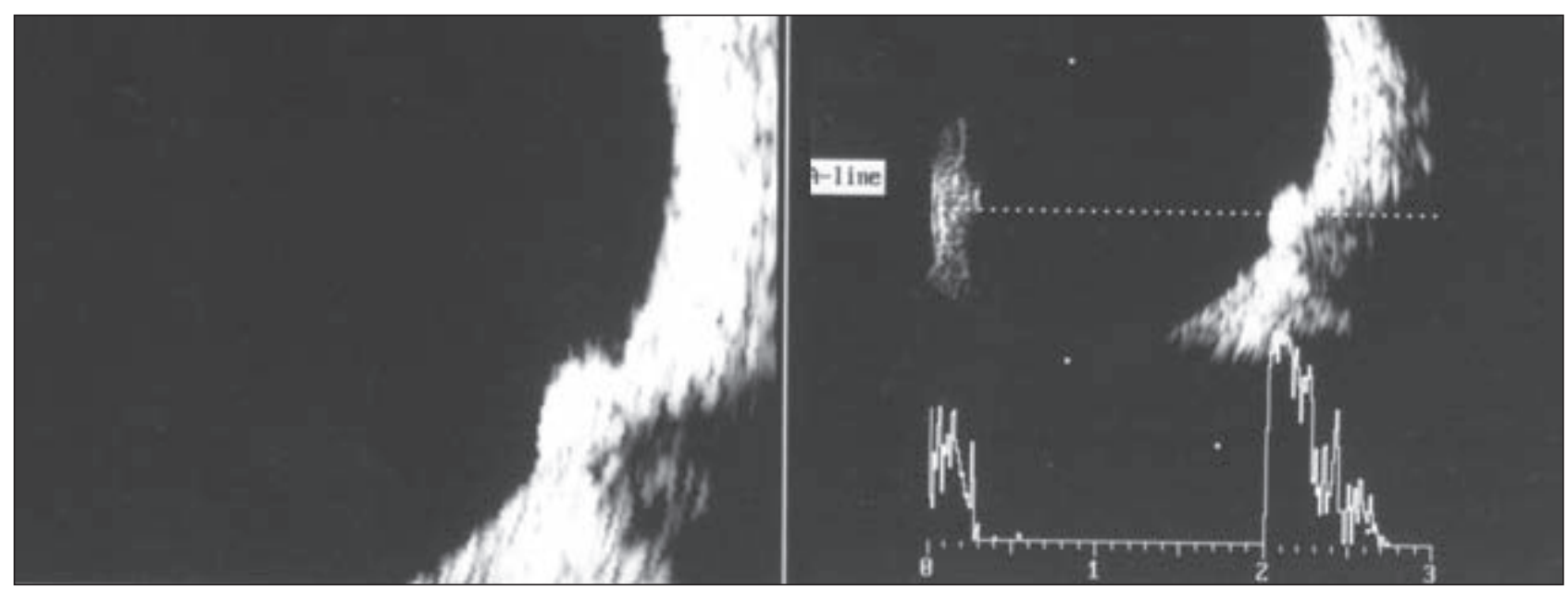

Figura 3: Ultra-som - Imagens revelam lesão sólida, cupuliforme, não móvel, em área papilar no meridiano das 4 à 6 horas, com refletividade interna heterogênea, média-alta sugestivo de melanocitoma

subjacentes, mostrando ter o melanoma um padrão sutil de diferença da coróide normal ${ }^{(38-39)}$.

\section{Exames complementares}

Os pacientes com suspeita de melanocitoma deveriam ser avaliados a partir da sua história clínica, exame oftalmológico completo, em particular a avaliação da pupila, complementando com a campimetria computadorizada, ultra-sonografia (modo A e B), retinografia, angiofluoresceinografia e mais recentemente a tomografia de coerência óptica ampliando assim o leque de exames para uma melhor avaliação diagnóstica e seguimento e garantindo aos pacientes portadores de melanocitoma a natureza benigna deste tumor.

\section{Angiofluoresceinografia}

Na maioria dos casos o estudo angiográfico do melanocitoma do disco óptico demonstra lesão com hipofluorescência precoce que se mantém durante todo exame ${ }^{(9-19)}$. Isto ocorre devido às células densamente pigmentadas e compactas, com pouca vascularização. Em casos de edema do disco óptico, temos hiperfluorescência da porção edemaciada do disco óptico (Figura 2).

\section{Campimetria computadorizada}

O melanocitoma do disco óptico pode causar uma variedade de defeitos no campo visual ${ }^{(40)}$. Osher e colaboradores ${ }^{(8)}$ mostraram que $90 \%$ dos pacientes apresentavam defeito do campo visual.As anormalidades incluíam aumento mínimo da mancha cega em (15\%), aumento severo da 

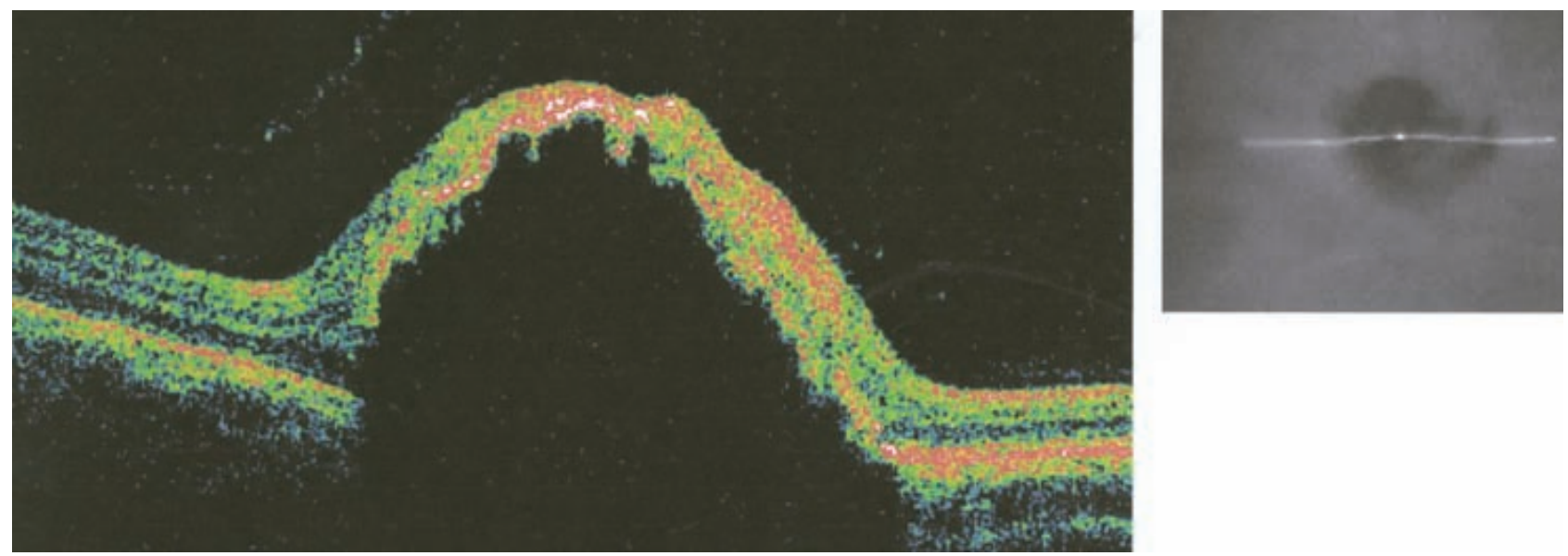

Figura 4: Tomografia de coerência óptica (OCT) mostrou uma lesão estendendo-se anteriormente com uma camada de alta-refletividade, com sombreamento atrás deste, representando o melanocitoma, obscurecendo todos os detalhes do disco óptico e retina adjacente

mancha cega em $(75 \%)$, dos quais $(10 \%)$ apresentavam concomitante defeito das fibras nervosas incluindo degrau nasal, defeito relativo na camada de fibras nervosas (20\%) e defeito arqueado absoluto em (20\%). O aumento da mancha cega parece estar relacionado à extensão do tumor, além da margem do disco óptico e o defeito arqueado está relacionado à compressão dos axônios do disco óptico ${ }^{(29)}$.

A realização do exame de ultra-som é extremamente valiosa auxiliando no diagnóstico. A característica mais comum do melanocitoma é o seu crescimento lento, permitindo acompanhar a pequena variação do tamanho através do exame de ultra-som modo A e B. O modo A é o método mais acurado para mensuração do tamanho da lesão que raramente protrunde mais do que 1 a $2 \mathrm{~mm}{ }^{(27)}$, já o modo B tipicamente apresenta solidez acústica com uma refletividade interna regular e predominantemente alta, sem vascularização interna ${ }^{(24,41,45)}$. $\mathrm{O}$ registro ultrassonográfico depende da arquitetura e densidade do tecido analisado. $\mathrm{O}$ aumento da refletividade interna e da atenuação sonora ocorre à medida que aumenta o tamanho do tumor ${ }^{(43)}$ (Figura 3).

\section{Tomografia de coerência óptica (oct)}

O exame de OCT é uma nova técnica que tem sido usado para avaliar o melanocitoma do disco óptico ${ }^{(46)}$. Embora, o melanocitoma não tenha uma apresentação específica ao OCT, a técnica aparenta ser útil em determinar a extensão do fluido sub-retiniano e edema de retina, achados que não são facilmente detectados com outros métodos (Figura 4).

\section{Tratamento}

Uma vez que o melanocitoma do disco óptico raramente sofre transformação maligna, exames e foto- grafias devem ser realizados anualmente.

A freqüência de seguimento do melanocitoma do disco óptico está na dependência das características do tumor. Se a lesão pigmentar é descoberta na primeira visita, o paciente deve ser questionado quanto à detecção de exames prévios e então ser realizados testes apropriados como citados anteriormente e retornar em um mês. Se não houver mudança da aparência da lesão, recomenda-se uma reavaliação em seis meses. Teste de campimetria deve ser repetido anualmente para monitorar perda de visão não detectada pelo crescimento aparente do tumor ${ }^{(4,41)}$.

Nos casos em que se confirme crescimento progressivo e perda visual sugerindo malignização devem se considerar a enucleação.

\section{Abstract}

Melanocytoma of the optic disc is a well known variant of melanocytic nevus that usually occurs as a deeply pigmented lesion on the head of the optic disc. Historically, this tumor has often been confused with malignant melanoma. Histopathologically, it is composed of deeply pigmented round oval cells with abundant cytoplasm and small, round, bland nuclei. Melanocytomas is a benign, stationary tumors, with almost no propensity to undergo malignant transformation. Most cases that occur on the optic disc are visually asymptomatic, but they can cause an afferent pupillary and visual field defects. Importantly, it can exhibit malignant transformation into melanoma in 1 to $2 \%$ of the cases. The affected patients should have periodic follow up.

Keywords: Melanoma/diagnosis; Optic disk/ pathology; Optic nerve neoplasms/pathology; Melanocytes/pahology; Diagnosis, differential; Review 


\section{REFERÊNCIAS}

1. Thomas CI, Purnell EW. Ocular melanocytoma. Am J Ophthalmol. 1969; 67(1):79-86.

2. Barker-Griffith AE, McDonald PR, Green WR. Malignant melanoma arising in a choroidal magnacellular nevus (melanocytoma). Can J Ophthalmol. 1976; 11(2):140-6.

3. Roth AM. Malignant change in melanocytomas of the uveal tract. Surv Ophthalmol. 1978; 22 (6):404-12.

4. Zimmerman LE, Garron L. Melanocytoma of the optic disc. Int Ophthalmol Clin 1962; 2:431-4.

5. Zimmerman LE. Melanocytes, melanocytic nevi, and melanocytomas. Invest Ophthalmol. 1965; 4:11-41.

6. Zimmerman LE. Changing concepts concerning the malignancy of ocular tumors. Arch Ophthalmol. 1967; 78(2):166-73.

7. Loewenstein A. Aberrant optic nerve fibres found between retina and hexagonal cells. Br J ophthalmol. 1945; 29(4):180-5.

8. Osher RH, Shields JA, Layman PR. Pupillary and visual field evaluation in patients with melanocytoma of the optic disc. Arch Ophthalmol. 1979; 97(6):1096-9.

9. Shields JA, Demirci H, Mashayekhi A, Shields CL. Melanocytoma of optic disc in 115 cases: the 2004 Samuel Johnson Memorial Lecture, part 1. Ophthalmology. 2004; 111(9):1739-46.

10. Shields JA, Shields CL, Piccone M, Snady-McCoy LC. Spontaneous appearance of an optic disk melanocytoma in an adult. Am J Ophthalmol. 2002; 134(4):614-5.

11. Shields JA, Annesley WH Jr, Spaeth GL. Necrotic melanocytoma of iris with secondary glaucoma. Am J Ophthalmol. 1977 Dec; 84(6):826-9.

12. Shields JA, Font RL. Melanocytoma of the choroid clinically simulating a malignant melanoma. Arch Ophthalmol. 1972; 87(4):396-400.

13. Lee JS, Smith RE, Minckler DS. Scleral melanocytoma. Ophthalmology. 1982; 89(2):178-82.

14. Verdaguer J, Valenzuela H, Strozzi L. Melanocytoma of the conjunctiva. Arch Ophthalmol. 1974;91(5):363-6.

15. Frangieh GT, el Baba F, Traboulsi EI, Green WR. Melanocytoma of the ciliary body: presentation of four cases and review of nineteen reports. Surv Ophthalmol. 1985; 29(5):328-34.

16. Stokes DW, O'Day DM, Glick AD. Melanocytoma of the ciliary body with scleral extension. Ophthalmic Surg. 1993; 24(3):200-2.

17. Marback RL, Sé DCS, Barsante C. Tumores da papila e do nervo óptico. In: Abujamra S editor. Retina e vítreo clínica e cirurgia. São Paulo: Roca; 2000. p.387-91.

18. Shields JA. Diagnosis and management of intraocular tumors. St. Louis: Mosby; 1983.

19. Shields JA, Shields CL. Intraocular tumors: a text and atlas. Philadelphia: WB Saunders; 1992. Melanocytoma; p.101-15.

20. Shields JA, Shields CL. Atlas of Intraocular Tumors. Philadelphia: Lippincott Williams and Williams; 1999. Melanocytoma; p.324-5.

21. Juarez CP, Tso MO. An ultrastructural study of melanocytomas (magnocellular nevi) of the optic disk and uvea. Am J Ophthalmol. 1980; 90(1):48-62.

22. Sharma PM, Sangal K, Malik P, Mathur MB. Malignant transformation of optic disc melanocytoma? A clinical dilemma at presentation with a review of the literature. Ophthalmologica. 2002;216(4):292-5.

23. Mansour AM, Zimmerman L, La Piana FG, Beauchamp GR. Clinicopathological findings in a growing optic nerve melanocytoma. Br J Ophthalmol. 1989; 73(6):410-5.

24. Shields JA, Shields CL, Eagle RC Jr, Lieb WE, Stern S. Malignant melanoma associated with melanocytoma of the optic disc. Ophthalmology. 1990; 97(2):225-30.

25. Apple DJ, Craythorn JM, Reidy JJ, Steinmetz RL, Brady SE, Bohart WA. Malignant transformation of an optic nerve melanocytoma. Can J Ophthalmol. 1984; 19(7):320-5.

26. Joffe L, Shields JA, Osher RH, Gass JD. Clinical and followup studies of melanocytomas of the optic disc. Ophthalmology. 1979; 86(6):1067-83.
27. Wiznia RA, Price J. Recovery of vision in association with a melanocytoma of the optic disk. Am J Ophthalmol. 1974;78(2):236-8.

28. Brodsky MC, Phillips PH. Optic nerve hypoplasia and congenital hypopituitarism. J Pediatr. 2000; 136(6):850.

29. Reidy JJ, Apple DJ, Steinmetz RL, Craythorn JM, Loftfield K, Gieser SC, et al. Melanocytoma: nomenclature, pathogenesis, natural history and treatment. Surv Ophthalmol. 1985; 29(5):319-27.

30. Albert DM, Jakobiec FA. Nevus and melanocytoma. In: Sahel JA, Meyer LH, Marcus DM, Albert DM. Principles and practice of ophthalmology Philadelphia: Saunders; 1994. p. 5057-64.

31. Schachat AP, Shields JA, Fine SL, Sanborn GE, Weingeist TA, Valenzuela RE, et al. Combined hamartomas of the retina and retinal pigment epithelium. Ophthalmology. 1984; 91(12):1609-15.

32. Shields JA, Eagle RC Jr, Barr CC, Shields CL, Jones DE. Adenocarcinoma of retinal pigment epithelium arising from a juxtapapillary histoplasmosis scar. Arch Ophthalmol. 1994; 112(5):650-3.

33. Shields JA, Eagle RC Jr, Shields CL, De Potter P. Pigmented adenoma of the optic nerve head simulating a melanocytoma. Ophthalmology. 1992; 99(11):1705-8.

34. Shields JA, Shields CL, Singh AD. Metastatic neoplasms in the optic disc: the 1999 Bjerrum Lecture: part 2. Arch Ophthalmol. 2000; 118(2):217-24.

35. De Potter P, Shields CL, Eagle RC Jr, Shields JA, Lipkowitz JL. Malignant melanoma of the optic nerve. Arch Ophthalmol. 1996; 114(5):608-12.

36. Erzurum SA, Jampol LM, Territo C, O'Grady R. Primary malignant melanoma of the optic nerve simulating a melanocytoma. Arch Ophthalmol. 1992; 110(5):684-6.

37. Shields CA, Shields JA. Tumores intra-oculares. In:Vilela MAP, editor. Angiografia fluoresceínica - Atlas \& Texto. Rio de Janeiro: Cultura Médica; 2005. p.139-40.

38. Schaudig U, Hassenstein A, Bernd A, Walter A, Richard G. Limitations of imaging choroidal tumors in vivo by optical coherence tomography. Graefes Arch Clin Exp Ophthalmol. 1998; 236(8):588-92.

39. Fujimoto JG, Pitris C, Boppart SA, Brezinski ME. Optical coherence tomography: an emerging technology for biomedical imaging and optical biopsy. Neoplasia. 2000; 2(1-2):9-25.

40. Meyer D, Ge J, Blinder KJ, Sinard J, Xu S. Malignant transformation of an optic disk melanocytoma. Am J Ophthalmol. 1999; 127(6):710-4.

41. Gupta V, Gupta A, Dogra MR, Pandav SS. Progressive growth in melanocytoma of the optic nerve head. Indian $\mathbf{J}$ Ophthalmol. 1995; 43(4):198-200.

42. Chojniak MM. Ultra-som nos tumores intra-oculares. In: José NK, editor. Ecografia ocular e orbitária. São Paulo: Roca; 2003.p.83-114.

43. Peyman GA, Sanders DR, Goldberg MF. Contact A-scan ultrasonography. In: Diamond JG, Ossoining CC editors. Principles and practice of ophthalmology. Philadelphia: Saunders; 1997. p.1405-88.

44. Di Bernardo C, Schachat AP, Fekrat S. Intraocular Tumors. In: DiBernardo CW, Schachat AP, Fekrat S, editors. Ophthalmic ultrasound: a diagnostic atlas. New York: Thieme; 1998. p.87-107.

45. Byrne SF, Green RL. Intraocular tumors. In: Byrne SF, Green RL, editors. Ultrasound of the eye and orbit. St. Louis: Mosby; 1992. p.180-1.

46. Antcliff RJ, ffytche TJ, Shilling JS, Marshall J. Optical coherence tomography of melanocytoma. Am J Ophthalmol. 2000;130(6):845-7

\section{ENDEREÇO PARA CORRESPONDÊNCIA: \\ Enéias Bezerra Gouveia \\ Rua Cubatão, 1111 - apto 21 - Vila Mariana \\ CEP 04013-044 - São Paulo - SP \\ Email: baslerdolansky@uol.com.br}

